\title{
A Short and Elementary Proof of the Two-sidedness of the Matrix-Inverse
}

Pietro Paparella (pietrop@uw.edu) University of Washington Bothell

Beauregard [2] and Strang [7, pp. 88-89] give an elementary proof of the twosidedness of the matrix-inverse using elementary matrices. Sandomierski [6] gives another elementary proof using an under-determined homogeneous linear system. Hill [3] provides a proof using basic results of rings and ideals, and Baksalary \& Trenkler [1] provide a proof using the Moore-Penrose inverse.

However, as we shall see, the two-sidedness of the matrix inverse can be shown using only the notions of linear independence and the reduced row-echelon form of a matrix, concepts that feature prominently in an elementary linear algebra course (it is worth noting that the proof of Theorem 1 is in the same vein as that found in the advanced textbook by Meyer [5, Example 3.7.2]).

In addition, and as an application of this result, we prove that a matrix is invertible if and only if it is row-equivalent to the identity matrix without appealing to elementary matrices, which is common in textbooks (see, e.g., [4, 7]). This result is shown by utilizing the feasibility of certain linear systems and appealing to a well-known theorem.

Such demonstrations are valuable pedagogically, given that the linear system is the central object of study in a typical elementary linear algebra course.

\section{The Proofs}

In what follows all matrices are assumed to be $n$-by- $n$ and all vectors are $n$-by- 1 . As is standard, the identity matrix is denoted by $I$.

Theorem 1. If $A$ and $B$ are matrices such that $A B=I$, then $B A=I$.

Proof. First, we show that the columns of $B$ form a linearly independent set; to the contrary, if $B x=0$, where $x \neq 0$, then $x=I x=A B x=A(B x)=A(0)=0$, a contradiction.

Because the columns of $B$ form a linearly independent set, it follows that the reduced row-echelon form of $B$ contains a pivot in every column and hence every row; following a well-known theorem [4. Theorem 4, $\S 1.4]$, the equation $B x=b$ has a solution for every vector $b$.

Next, we argue that the columns of $A$ must form a linearly independent set; to that end, suppose $A y=0$, where $y \neq 0$. From previous, there is a necessarily nonzero vector $x$ such that $y=B x$. Thus, $0=A y=A(B x)=(A B) x=I x=x$, a contradiction.

Multiplying both sides of the equation $A B=I$ on the right by $A$ yields $A B A=$ $A$. Subtracting $A$ from both sides and applying the distributive property yields $A(B A-I)=0$.

We claim that the matrix $Z:=B A-I=0$; if not, then $Z$ contains a nonzero entry and hence a nonzero column - say the $j^{\text {th }}$-column, which we denote by $z_{j}$. Thus, $A z_{j}=0$, which is a contradiction unless $Z=B A-I=0$, i.e., $B A=I$.

Theorem 2. A matrix $A$ is invertible if and only if $A$ is row-equivalent to $I$.

Proof. Following Theorem 1, $A$ is invertible if and only if there is a matrix $X$ such that $A X=I$. Thus, $A$ is invertible if and only if the linear system $A x=e_{i}$ is feasible for all $i \in\{1, \ldots, n\}$, where $e_{i}$ denotes the $i^{\text {th }}$ column of $I$. 
We claim that if $A x=e_{i}$ is feasible for all $i \in\{1, \ldots, n\}$, then $A x=b$ is feasible for every vector $b$ (note that the converse of this statement is obviously true). To that end, if $s_{i}$ is any solution to $A x=e_{i}$ and $b=\left[b_{1} \cdots b_{n}\right]^{\top}$, then, following the linearity of $A$,

$$
b=I b:=\sum_{i=1}^{n} b_{i} e_{i}=\sum_{i=1}^{n} b_{i}\left(A s_{i}\right)=\sum_{i=1}^{n} A\left(b_{i} s_{i}\right)=A\left(\sum_{i=1}^{n} b_{i} s_{i}\right),
$$

and the claim is established.

Since $A x=b$ for every vector $b$, another application of [4, Theorem $4, \S 1.4$ ] reveals that $A$ is invertible if and only if the reduced row-echelon form of $A$ possesses a pivot in every row, i.e., $A$ is invertible if and only if it is row-equivalent to $I$.

Remark. Our demonstration proves the equivalence of the following statements of the invertible matrix theorem:

- $A$ is invertible.

- $A$ is row-equivalent to $I$.

- $A x=b$ is feasible for every vector $b$.

- the reduced row-echelon form of $A$ has a pivot in every row.

- the columns of $A$ are linearly independent.

- the equation $A x=0$ has only the trivial solution.

- the columns of $A$ span $\mathbb{R}^{n}$.

- the columns of $A$ form a basis for $\mathbb{R}^{n}$.

Summary. An elementary proof of the two-sidedness of the matrix-inverse is given using only linear independence and the reduced row-echelon form of a matrix. In addition, it is shown that a matrix is invertible if and only if it is row-equivalent to the identity matrix without appealing to elementary matrices. This proof underscores the importance of a basis and provides a proof of the invertible matrix theorem.

\section{References}

1. O. M. Baksalary and G. Trenkler, Another Proof of the Two-sidedness of Matrix Inverses, IMAGE, 50 (2013) 2 .

2. R. A. Beauregard, A short proof of the two-sidedness of matrix inverses, Math. Mag. 80 (2) (2007) 135-136.

3. P. Hill, On the matrix equation $A B=I$, American Math. Montly 74 (7) (1967) 848-849. DOI: $10.2307 / 2315819$

4. D. C. Lay, S. R. Lay, and J. J. McDonald, Linear Algebra and Its Applications, 5th ed., Pearson Education, 2016.

5. C. D. Meyer, Matrix Analysis and Applied Linear Algebra, SIAM, Philadelphia, PA, 2000.

6. F. Sandomierski, An elementary proof of the two-sidedness of matrix inverses, Math. Mag. 85 (4) (2012) 289. DOI: $10.4169 /$ math.mag. 85.4 .289

7. W. G. Strang, Introduction to Linear Algebra, 5th ed., Wellesley-Cambridge, Wellesley, MA, 2016. 